\title{
Acceptability of tick control interventions to prevent Lyme disease in Switzerland and Canada: a mixed-method study
}

\author{
Cécile Aenishaenslin ${ }^{1,6^{*}}$, Pascal Michel ${ }^{1,2}$, André Ravel ${ }^{6}$, Lise Gern ${ }^{3}$, Jean-Philippe Waaub ${ }^{5}$, François Milord ${ }^{4}$ \\ and Denise Bélanger ${ }^{1,6}$
}

\begin{abstract}
Background: Lyme disease control strategies may include tick control interventions in high risk areas. Public authorities may be interested to assess how these types of interventions are perceived by the public which may then impact their acceptability. The aims of this paper are to compare socio-cognitive factors associated with high acceptability of tick control interventions and to describe perceived issues that may explain their low acceptability in populations living in two different regions, one being an endemic region for LD since the last 30 years, the Neuchâtel canton, in Switzerland, and another where the disease is emerging, the Montérégie region, in Canada.

Methods: A mixed methods' design was chosen. Quantitative data were collected using web-surveys conducted in both regions $(n=814)$. Multivariable logistic regressions were used to compare socio-cognitive factors associated with high acceptability of selected interventions. Qualitative data were collected using focus group's discussions to describe perceived issues relative to these interventions.

Results: Levels of acceptability in the studied populations were the lowest for the use of acaricides and landscaping and were under $50 \%$ in both regions for six out of eight interventions, but were higher overall in Montérégie. High perceived efficacy of the intervention was strongly associated with high acceptability of tick control interventions. A high perceived risk about LD was also associated with a high acceptability of intervention under some models. High level of knowledge about LD was negatively associated with high acceptability of the use of acaricides in Neuchâtel. Perceived issues explaining low acceptability included environmental impacts, high costs to the public system, danger of individual disempowerment and perceptions that tick control interventions were disproportionate options for the level of LD risk.

Conclusion: This study suggests that the perceived efficacy and LD risk perception may be key factors to target to increase the acceptability of tick control interventions. Community-level issues seem to be important considerations driving low acceptability of public health interventions. Results of this study highlight the importance for decision-makers to account for socio-cognitive factors and perceived issues that may affect the acceptability of public health interventions in order to maximize the efficacy of actions to prevent and control LD.
\end{abstract}

Keywords: Tick control, Lyme disease, Borreliosis, Prevention, Acceptability, Risk perception, Mixed-methods, Intervention acceptability

\footnotetext{
* Correspondence: cecile.aenishaenslin@umontreal.ca

'Research Group on Epidemiology of Zoonoses and Public Health, Pavillon

de la santé publique, Faculté de médecine vétérinaire, Université de

Montréal, CP 5000, Saint-Hyacinthe, J2S 7C6 Québec, Canada

${ }^{6}$ Département de pathologie et microbiologie, Faculté de médecine

vétérinaire, Université de Montréal, CP 5000, Saint-Hyacinthe, J2S 7C6

Québec, Canada

Full list of author information is available at the end of the article
} 


\section{Background}

Lyme disease (LD) is the most frequently reported vector-borne disease in the temperate countries. Caused by the bacteria Borrelia burgdorferi and transmitted to humans by a tick bite, the incidence of this multisystemic disease is increasing in several regions around the world [1-3]. In the absence of a vaccine, preventive strategies adopted by affected countries mostly target the promotion of individual preventive behaviors against tick bites such as wearing protective clothing, applying tick repellent on skin and clothing, checking for and removing ticks after visiting wooded areas, and avoiding tick habitats during high-risk periods [4]. Several studies demonstrated that these behaviors were effective to protect oneself against LD [4-10]. Nevertheless, few studies also underlined that even in regions where LD incidence is high and where the population had a good level of knowledge about the disease, the proportion of people that effectively adopted these behaviors could be quite low, and that these different behaviors were not adopted with the same success by the individuals [11-16]. Tick control interventions have been studied as well as complementary strategies to prevent LD (reviewed in Piesman and Eisen [4]). Studies have shown that these interventions could effectively decrease the vector density in risk areas for LD. These strategies include direct actions on tick populations such as the use of acaricides [17], landscaping [18-20] or biological control of ticks [21], and actions that target wild animal species, which are the main hosts of the vector or the reservoirs of the agent, such as the reduction of deer density [22], the treatment of deer against ticks [23-25], the treatment of small rodents against ticks [26-30] and the vaccination of rodents against Borrelia sp. [31-34]. But such interventions used by public health authorities can have true or perceived impacts that may affect the acceptability of these choices in the targeted population and can cause controversy, such as their negative consequences for the environment or their adverse health effects $[35,36]$. One example of this kind of debate experimented in Canada was caused by the use of larvicide in order to reduce mosquito populations and the risk of West Nile Virus infection [37]. Regarding $\mathrm{LD}$, a previous study reported that stakeholders involved in decision-making toward LD prevention in Canada and Switzerland identified social acceptability as an important decision criterion to prioritize preventive interventions $[38,39]$. In policy research, it is well known that public opinion has an impact on policy choices (38). But despite its recognized importance, very few published studies reported empirical data on the acceptability of interventions to prevent and control LD. Gould and colleagues [40] measured the acceptability of three environmental interventions against LD in three districts in Connecticut, United States, during two different periods. Their study suggested that the level of acceptability changes according to the intervention, the region of residency and through time. Populations living in districts with the higher LD incidence showed higher levels of acceptability of tick control interventions, but no formal statistical tests were applied to confirm this relationship [40]. To our knowledge, no published study explored factors that may be associated with acceptability of these interventions, nor what could explain low acceptability of some interventions in different populations. A better understanding of these factors would be an important addition to the current knowledge on the efficacy of these interventions and would be of great use to enhance informed decisionmaking by public health authorities.

The aims of this paper are 1) to measure and compare levels of acceptability of tick control interventions, as well as their associated socio-cognitive factors, and 2) to describe perceived issues that may explain their low acceptability in populations living in two different regions, one being an endemic region for LD since the last 30 years, the Neuchâtel canton, in Switzerland, and another where the disease is emerging, the Montérégie region, in Québec, Canada [3]. The choice of these two contrasting regions in terms of their epidemiological situation toward LD gives the opportunity to explore more specifically the influence of the epidemiological situation, and of some socio-cognitive factors related to different contexts on acceptability, that are the level of knowledge on LD in the population and the level of risk perception. Aenishaenslin and colleagues have shown that levels of knowledge and risk perception about LD were higher in Neuchâtel than in Montérégie [41]. In this context, we hypothesized that tick control interventions are more acceptable in regions with higher risk of LD (here in Neuchâtel), than in regions with lower risk (Montérégie). For this study, we defined the notion of acceptability as a self-reported positive attitude of individuals toward a hypothetical intervention, given that none of the tick control interventions evaluated in this study had been implemented in both regions before data collection.

\section{Methods}

A mixed methods' design was undertaken for this comparative study. In both study regions, quantitative data were collected to measure levels of acceptability for different interventions as well as their potential associated factors, and qualitative data were collected concurrently to identify and describe perceived issues that may explain the low acceptability of the least accepted interventions. Results of both qualitative and quantitative analysis were interpreted together as complementary approaches to answer the study objectives. Informed written consent was obtained from all participants. This procedure 
and the study protocol were reviewed by the ethical committee for health research of the University of Montreal (Comité d'éthique de la recherche en santé, CERES) (certificate number 12-050-CERES-D), and the ethical certificate was approved by the Université de Neuchâtel.

\section{Quantitative data \\ Data collection}

Quantitative data were collected using cross-sectional web-surveys conducted simultaneously in fall 2012 in both study regions, the Montérégie region $(n=401)$ and the Neuchatel region $(n=413)$, as part of a larger study $[11,41]$. Respondents were selected randomly in representative web panels managed in each country by Léger and Marketing survey firm [42]. The complete questionnaire was administered in French and included 58 questions (available in [11]). Sixteen were specifically designed to measure levels of acceptability and levels of perceived efficacy of eight tick control interventions for LD in both studied populations, namely: using biologic control methods to reduce tick density, for example, the introduction of mushrooms capable of killing ticks (biological control), applying acaricides on public areas (acaricides), removing vegetation in public wooden areas (landscaping), protecting deer against ticks (deer protection), protecting rodents against ticks (rodent protection), vaccinating rodents against LD (rodent vaccination), controlling the number of deer (deer reduction) and excluding deer from public areas by fencing (fencing). Five-points Likert's scales were used as measurement units to assess the acceptability levels: "How acceptable do you think this intervention is to control LD with respect to your values? (5) strongly acceptable, (4) somewhat acceptable, (3) neither acceptable or unacceptable, (2) somewhat unacceptable, (1) strongly unacceptable; as well as the levels of perceived efficacy: "Do you agree that this intervention is effective to reduce the risk of LD?": (5) strongly agree, (4) somewhat agree, (3) neither agree nor disagree, (2) somewhat disagree, (1) strongly disagree." Additional data collected and used in this study included: gender, age, education level, level of knowledge on LD, and the level of risk perception for LD. Details on the survey design and on data collection strategies are described in more detail in Aenishaenslin et al. [41].

\section{Data analysis}

Descriptive and multivariable statistical analyses were conducted. For the descriptive analyses, we calculated the proportions of respondents with high acceptability (individual scores of 4 or 5 on the Likert's scale) for each of the eight interventions by region. Confidence intervals for proportions (confidence level of $95 \%$ ) were calculated using Agresti-Coull method with R software [43]. Pearson Chi-square statistics were calculated to assess significant differences $(p<0.05)$ between regions. This was done to evaluate the levels of acceptability for each intervention in both populations.

For the multivariable analysis, eight multivariable logistic regression models were built: one per region for each of these four interventions: landscaping, acaricide, rodent vaccination and fencing. These four interventions were selected for multivariable analyses because they were the interventions with the lowest levels of acceptability in both populations according to the descriptive analyses. This was done to study the relationships of three specific socio-cognitive factors: knowledge, risk perception, and perceived efficacy, on high acceptability of these interventions at the individual level. Dependent variables were the acceptability of the interventions (low $v s$ high), dichotomized as 'low' acceptability (individual scores of 1 to 3), and 'high' acceptability (individual scores of 4 or 5 ). Independent variables were 1 ) the global LD knowledge level, 2) the global risk perception score, and 3) the perceived efficacy of the specific control interventions. The global LD knowledge level was dichotomized in low if 0 to 2 good answers, and high if 3 or 4 good answers, based on four LD knowledge related questions about the transmission mode, early symptoms, treatment, and risk zones [41]. The global risk perception score was the calculated mean score of four observed perception variables about the disease (perceived severity, perceived individual susceptibility, perceived regional susceptibility and feelings of worry, as described in Aenishaenslin et al. [41]) and was considered as a continuous variable in the multivariable analyses. The perceived efficacy of the specific control interventions was also dichotomized in low (scores 1 to 3 ) and high (scores 4 and 5) in the models. Gender, age and education level were considered as potential confounders and forced in all models. Only statistically significant associations are shown for these variables. Participants with missing values ("prefer not to answer") for one of the included variables were excluded from the models (Montérégie: $n=8$; Neuchâtel, $n=6$ ). Multivariable analyses were performed using IBM SPSS Statistics 19.

\section{Qualitative data \\ Data collection}

Qualitative data were collected during five focus group discussions (FGD) conducted between August and October 2012 (previously to the websurveys) in Neuchâtel city and La-Chaux-de Fond (Neuchâtel canton, Switzerland) and in Saint-Hyacinthe and Longueuil (Montérégie region, Québec, Canada). In each region, residents who were more than 18 years old were invited to participate. Recruitment of this convenience sample was done locally through posters displayed in grocery stores, train stations, universities, public parks and health centers. 
Advertisements were also posted on outdoor group websites and sent to their members via e-mail. Specific questions addressed were: "Since when did you know LD? What worries you about this disease? What do you think about these interventions to prevent LD? Are they acceptable according to you and why?". Four environmental interventions were specifically addressed during FGD, based on their anticipated low level of acceptability in the targeted populations: landscaping, acaricides, rodent vaccination and fencing. Basic information on the interventions was given by the moderator, and participants were welcome to ask complementary questions if needed to allow a common understanding of the interventions. All participants also completed the questionnaire used in the websurveys prior to the discussions. The data collected with these questionnaires were used to compute the descriptive characteristics of participants per region. FGD were conducted in French and lasted one and a half hour. They were recorded and then transcribed entirely.

\section{Data analysis}

A thematic analysis was performed by the principal researcher (Aenishaenslin C) with the objective to identify and describe the perceived issues that may contribute to the low acceptability of the four selected interventions $[44,45]$. Thematic analysis can be divided in six phases: familiarisation with the data, generating initial codes, searching for themes, reviewing themes, defining and naming themes and producing the report [44]. Records and transcripts were first used during the familiarisation phase of analysis. Then, transcripts were analysed to create initial codes representing categories of issues expressed by participants in an iterative process, using QRS NVivo version 10. Codes were then grouped into themes representing the perceived issues related to the acceptability of interventions. Themes were named and reviewed using original transcripts. Extract examples were finally selected in each region, based on their representability of the issue in question. Perceived issues relative to low acceptability were analysed globally rather than for each intervention in order to draw general observations. Details relative to the interviewer and to qualitative data collection, analyse and reporting are presented in the supplementary material, using the consolidated criteria for reporting qualitative studies (COREQ) [46].

\section{Results}

The proportions of respondents with high level of acceptability were significantly higher in Montérégie for all interventions (Fig. 1). The least accepted intervention was landscaping in both regions with $16 \%$ (66/ $401,95 \% \mathrm{CI}=13-20)$ in Montérégie and $10 \%$ (41/ $413,95 \% \mathrm{CI}=7-13$ ) in Neuchâtel (Pearson Chisquare $=7.60, p=0.006$ ). Following by increasing order of acceptability and respectively in Montérégie and Neuchâtel: acaricides with 29 \% (116/401, 95 \% CI = 25-34) and $12 \%(51 / 413,95 \% \mathrm{CI}=9-16)$ (Pearson Chi-square $=34.29, p<0.001)$, rodent vaccination with $33 \%(131 / 401,95 \% \mathrm{CI}=28-37)$ and $26 \%(108 / 401$, $95 \% \mathrm{CI}=22-31$ ) (Pearson Chi-square $=4.17, p=0.04$ ), fencing with $37 \%(150 / 401,95 \% \mathrm{CI}=33-42)$ and $14 \%$ $(56 / 413,95 \% \mathrm{CI}=11-17)$ (Pearson Chi-square $=61.21$, $p<0.001)$, deer reduction with $47 \%(187 / 401,95 \%$ $\mathrm{CI}=42-52)$ and $31 \%(126 / 413,95 \% \mathrm{CI}=26-35)$ (Pearson Chi-square $=22.35, p<0.001)$, rodents protec tion with $51 \%(204 / 401,95 \% \mathrm{CI}=46-56)$ and $42 \%$ $(172 / 413,95 \% \mathrm{CI}=37-46)$ (Pearson Chi-square $=6.97$, $p=0.008)$, deer protection with $66 \%(266 / 401,95 \%$ $\mathrm{CI}=62-71)$ and $51 \%(211 / 413,95 \% \mathrm{CI}=46-56)$ (Pearson Chi-square $=19.49, p<0.001$ ), and biological control with $86 \%(345 / 401,95 \% \mathrm{CI}=82-89)$ and $76 \%$ $(315 / 413,95 \% \mathrm{CI}=72-80) \quad($ Pearson Chi-square $=12.64$, $p<0.001)$.

In the multivariable analyses, socio-cognitive factors that were statistically associated with high acceptability of landscaping, acaricides, rodent vaccination and fencing varied in both regions (Table 1). High perceived efficacy of the intervention was strongly associated with high acceptability of all interventions in all models. Risk perception was positively associated with high acceptability of landscaping in both regions, and it was positively associated with high acceptability of acaricides and fencing in Montérégie. High level of knowledge about LD was negatively associated with high acceptability of acaricides in Neuchâtel. Being between 18 and 34 years old was negatively associated with high acceptability of acaricides in Montérégie, in comparison to those who were more than 55 years old. Having a university degree was negatively associated with high acceptability of landscaping in Neuchâtel. When considering confidence intervals of OR (confidence level: $95 \%$ ), there was no difference regarding the strength of association between knowledge, risk perception and perceived efficacy and high acceptability of interventions between regional models.

Thirty-four participants were recruited for the five FGD (specific locations, number of participants and participant characteristics are presented in Table 2). Women represented $71 \%$ of the participants (24/34), and age groups from 18-24 years old to 75+ years old were represented by at least one participant. Most participants had a high level of knowledge about LD (58 \% in Montérégie and $86 \%$ in Neuchâtel). In Neuchâtel, 27 \% (7/22) of participants had a high risk perception score, compared to $50 \%$ (6/12) in Montérégie.

Through qualitative analysis, we identified eight common themes representing the perceived issues that may explain the low acceptability of the four selected interventions in the FGD performed in both regions. The 


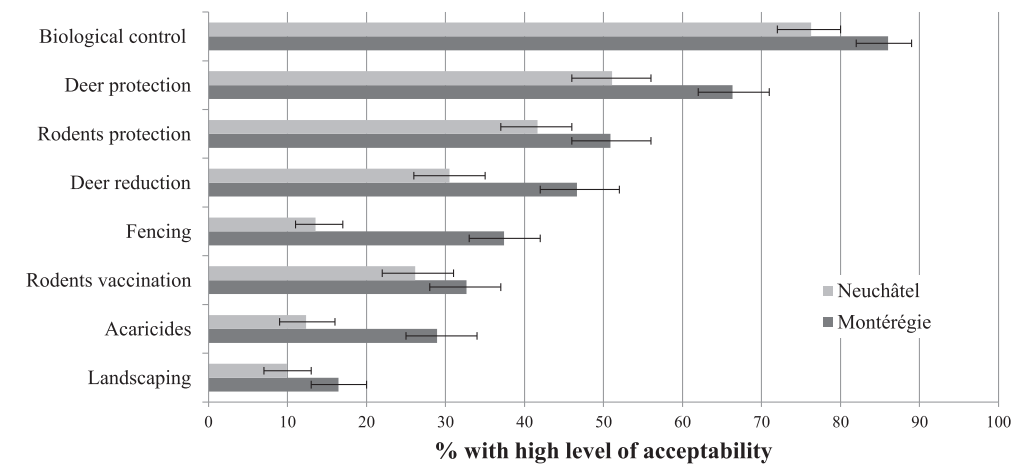

Fig. 1 Levels of acceptability of tick control interventions in Neuchâtel and Montérégie. This figure shows the proportions of respondents with high acceptability (scores of 4 or 5) for eight tick control interventions against LD in Neuchâtel $(n=413)$ and Montérégie $(n=401)$

recurrence of these themes into all focus groups suggests that a good level of data saturation was achieved. The first and commonly reported in both regions was the perception that the intervention would be disproportionate to manage the level of risk due to LD. Even with a good knowledge of LD and being aware that the risk of contracting the disease was present in their region, participants frequently mentioned that LD risk was not high enough for the population to justify the use of tick control interventions, as reported by a Montérégie participant: 'In the current situation, I do not feel it would be important to have such an impact, but in case it becomes a major public health problem, I would consider [rodent vaccination]'. In relation to this perception of disproportion, the fear of unknown consequences following the use of interventions to reduce the tick density was frequently mentioned, as illustrated by this Neuchâtel participant, while discussing about rodent vaccination: 'There is chemistry in this intervention. Maybe we eradicate an evil, but we recreate another'. More specifically, participants of both regions mentioned the fear of negative environmental impacts. This issue was both reported as a rational argument based on the potential impact on biodiversity or natural habitat: '[...] If we speak of destroying animal habitat in protected areas, it seems to me rather questionable ... it depends upon the impact on other species.' (Montérégie participant about landscaping). This fear was also expressed as a conflict with more general personal values regarding both animal well-being and protection of natural environments. This dimension was often mentioned in both regions, but more clearly defined in Neuchâtel: 'I do not see why we should change the nature because of a little bug' (Neuchâtel participant about landscaping). Another perception frequently mentioned was the economic impacts of implementing the interventions. Participants of both regions shared concerns about the high public costs of interventions, as stated by this Neuchâtel participant: '[...] To take rodents and vaccinate them... we can spend public money otherwise'.

Implementing these tick control interventions to prevent LD was also perceived as a threat to individual empowerment. It was perceived that if public health authorities made the choice of using such interventions, individuals would be tempted to distance themselves from their responsibility to adopt preventive behaviors: 'To want to protect humans by destroying nature when they do nothing to protect themselves, to me, it is unacceptable '(expressed by a Montérégie participant). Doubts about the feasibility of the interventions were also reported, regarding all presented interventions. Even after explanations from the FGD moderator about the feasibility of the interventions, participants had sometimes difficulties to believe that it was feasible, as stated by this Montérégie participant: 'I find it peculiar a little. How to prevent deer from going to places? [...] Can you really control it? I don't think that you can control the movement of deer.' Finally, some interventions, such as landscaping, were perceived as acceptable only if implemented at a smaller scale: It is also different concepts if it is to destroy half a forest to reduce the tick population [or] if it is to expand certain paths by removing a portion of the low vegetation [...]. That is acceptable because it has less impact on the ecosystem.' (Neuchâtel participant about landscaping).

We did not identify major differences through qualitative analysis in categories of issues explaining low acceptability of these tick control interventions between Neuchâtel and Montégérie participants. One observed difference was that Neuchâtel participants more frequently stated that public authorities should prioritize actions aiming to raise the population awareness on LD and promote individual preventive behaviors instead of considering the use of tick control interventions to reduce LD risk: 'I would go further. [Fencing] is indefensible. It is not always to the man to adapt the landscape to what he wants [...] It is also up to us to learn to live with our environment.' (Neuchâtel participant). Moreover, 
Table 1 Factors associated with high acceptability (scores of 4 or 5) of landscaping, acaricide, rodent vaccination and fencing (Logistic regressions)

\begin{tabular}{llllll}
\hline \multicolumn{1}{l}{ Factors associated with high acceptability of landscaping } \\
& Montérégie $(n=393)$ & \multicolumn{3}{l}{ Neuchâtel $(n=407)$} \\
& OR & $95 \% \mathrm{Cl}$ & OR & $95 \% \mathrm{Cl}$ \\
Age 18-34 years & 0.32 & $(0.09-1.10)$ & 0.51 & $(0.13-1.98)$ \\
$35-54$ years & 0.77 & $(0.39-1.54)$ & 2.70 & $(0.93-7.88)$ \\
$55+\mathrm{yr}^{\mathrm{R}}$ & 1 & $\mathrm{na}$ & 1 & $\mathrm{na}$ \\
Education (University) & 0.83 & $(0.39-1.76)$ & 0.36 & $(0.15-0.88)^{*}$ \\
Knowledge of LD & 1.02 & $(0.42-2.48)$ & 0.72 & $(0.31-1.67)$ \\
Risk perception & 1.64 & $(1.05-2.57)^{*}$ & 2.88 & $(1.45-5.74)^{* *}$ \\
Perceived efficacy & 16.31 & $(8.50-31.28)^{* * *}$ & 37.48 & $(13.95-100.71)^{* * *}$
\end{tabular}

Factors associated with high acceptability of the use of acaricides

\begin{tabular}{llllll} 
& \multicolumn{2}{l}{ Montérégie $(n=393)$} & \multicolumn{2}{l}{ Neuchâtel $(n=407)$} \\
& OR & $95 \% \mathrm{Cl}$ & OR & $95 \% \mathrm{Cl}$ \\
Age 18-34 years & 0.16 & $(0.05-0.49)^{* * *}$ & 0.47 & $(0.18-1.23)$ \\
35-54 years & 0.98 & $(0.54-1.80)$ & 0.85 & $(0.37-1.95)$ \\
55+ yr ${ }^{R}$ & 1 & na & 1 & na \\
Knowledge of LD & 0.47 & $(0.20-1.07)$ & 0.33 & $(0.16-0.69)^{* *}$ \\
Risk perception & 1.60 & $(1.08-2.36)^{*}$ & 1.48 & $(0.88-2.50)$ \\
Perceived efficacy & 20.89 & $(11.41-38.22)^{* * *}$ & 12.28 & $(6.03-24.99)^{* * *}$
\end{tabular}

Factors associated with high acceptability of rodent vaccination

\begin{tabular}{llllll} 
& \multicolumn{2}{l}{ Montérégie $(n=393)$} & \multicolumn{2}{l}{ Neuchâtel $(n=407)$} \\
& OR & $95 \% \mathrm{Cl}$ & OR & $95 \% \mathrm{Cl}$ \\
Education (University) & 2.01 & $(1.11-3.66)^{*}$ & 0.69 & $(0.39-1.20)$ \\
Knowledge of LD & 0.67 & $(0.32-1.42)$ & 1.05 & $(0.61-1.81)$ \\
Risk perception & 1.34 & $(0.92-1.96)$ & 1.35 & $(0.91-2.00)$ \\
Perceived efficacy & 21.37 & $(12.15-37.58)^{* * *}$ & 17.37 & $(9.69-31.15)^{* * *}$
\end{tabular}

Factors associated with high acceptability of fencing to restrict deer from public areas

\begin{tabular}{lllll} 
& \multicolumn{3}{l}{ Montérégie $(n=393)$} & \multicolumn{2}{l}{ Neuchâtel $(n=407)$} \\
& OR & $95 \% \mathrm{Cl}$ & OR & $95 \% \mathrm{Cl}$ \\
Age 18-34 years & 0.65 & $(0.27-1.57)$ & 1.48 & $(0.53-4.16)$ \\
$35-54$ years & 0.56 & $(0.31-1.03)$ & 2.60 & $(1.04-6.48)^{*}$ \\
$55+\mathrm{yr}^{\mathrm{R}}$ & 1 & $\mathrm{na}$ & 1 & $\mathrm{Na}$ \\
Knowledge of LD $^{*}$ & 0.64 & $(0.29-1.43)$ & 0.93 & $(0.48-1.79)$ \\
Risk perception & 1.65 & $(1.13-2.41)^{* *}$ & 1.07 & $(0.68-1.68)$ \\
Perceived efficacy & 22,82 & $(12.83-40.59)^{* * *}$ & 10,17 & $(5.27-19.63)^{* * *}$ \\
\hline * $^{*}<0.05$ * ** $^{*}<0.01$ * ** $^{*}<0.001$ & & &
\end{tabular}

the perception that individuals can and should protect themselves was stronger among participants of this region when compared to Montérégie, where participants more frequently asked questions on how to prevent Lyme disease in general. Nevertheless, it was perceived that access to information about LD was lacking in both regions.

\section{Discussion}

This study showed that the levels of acceptability of tick control interventions to prevent LD was low in Neuchâtel and Montérégie, with only two interventions out of eight (deer protection and biological control) reaching at least $50 \%$ of the studied populations in both regions. These results contrast with those reported by Gould and colleagues in Connecticut (USA) [40]. In this study, levels of acceptability for deer control, deer protection, and acaricides were measured at two periods in 1999 and in 2004. All three interventions reached levels of acceptability of more than $50 \%$ of the population for the two periods and in all three studied districts [40]. One partial explanation for this difference can be that the incidence of LD in the studied districts ranged from 240 to 411 cases per 100,000 people at the time of the study, which is considerably higher than our two study regions [40]. Considering our results, the fact that respondents from Montérégie region in Canada had low levels of acceptability can be understood when considering that LD incidence in this region is still very low (estimated at 0.5 case per 100,000 inhabitants in Montérégie in 2012) [47]. The population may not perceive the need for these kinds of interventions. This explanation is corroborated by the qualitative analysis, underlining that one major issue related to low acceptability was the perception that tick control interventions are disproportionate options for the level of LD risk to which the population is exposed.

Surprisingly, the fact that levels of acceptability of all interventions were even lower in Neuchâtel canton was unexpected, given our initial hypothesis which stated that populations living in regions with higher incidence of LD would be more receptive to tick control interventions. Neuchâtel canton has an incidence estimated to be between 49 to 95 cases per 100,000 inhabitants per year (data from 1996 and 2001) [48, 49] which is still lower than the three Connecticut districts studied in Gould et al. [40], but much higher than in Montérégie, and comparable to some endemic regions of the East coast of the United States. Even if we cannot consider that FGD participants are representative of the surveyed population, results of the qualitative analysis provided information that may help to explain these findings: Neuchâtel participants seemed to put a high value on the environment and its conservation, and shared the perception that the risk of LD does not justify the use of interventions that can affect the environment. This is coherent with a recent study by Aenishaenslin and colleagues [39] which reported that Swiss stakeholders involved in decision-making for implementing LD preventive strategies did not want to include as hypothetical 
Table 2 Distribution of participants by region for the five FGD

\begin{tabular}{|c|c|c|}
\hline Region & Focus groups (number of participants) & Descriptive characteristics of participants by region \\
\hline \multirow[t]{7}{*}{ Neuchâtel } & Focus group 1 (7) & $22 / 22(100 \%)$ were aware of LD for more than one year \\
\hline & Focus group 2 (9) & 7/22 (27 \%) had a high risk perception (global risk perception score $\geq 4$ ) \\
\hline & Focus group 3 (6) & 19/22 (86 \%) had a high level of knowledge on LD \\
\hline & Total $=22$ & $3 / 22(14 \%)$ declared that they had LD in the past \\
\hline & & $17 / 22$ (77 \%) declared that they knew someone who has ever had LD \\
\hline & & 16/22 (73\%) were women \\
\hline & & Age of participants was distributed between 18 and more than 75 years old \\
\hline \multirow[t]{7}{*}{ Montérégie } & Focus group 4 (6) & $10 / 12(83 \%)$ were aware of LD for more than one year \\
\hline & Focus group 5 (6) & 6/12 (50 \%) had a high risk perception (global risk perception score $\geq 4$ ) \\
\hline & Total $=12$ & 7/12 (58 \%) had a high level of knowledge on LD \\
\hline & & $0 / 12(0 \%)$ declared that they had LD in the past \\
\hline & & 1/12 (8 \%) declared that they knew someone who has ever had LD \\
\hline & & $8 / 12(67 \%)$ were women \\
\hline & & Age of participants was distributed between 25 and 64 years old \\
\hline
\end{tabular}

interventions in a decision-model some tick control interventions such as application of acaricides or landscaping at large scales, because their potential impact on the environment was against their population's values.

Results from multivariable analyses showed that high perceived efficacy was strongly associated with high acceptability of interventions, and that risk perception was also a factor associated with it in several logistic models. These two socio-cognitive factors were associated with the adoption of individual preventive behaviors toward LD in a recent study which analyzed the same sampled populations [11], and they are known predictors of health behaviors in a number of theoretical models, including the Health Belief Model [50]. On the other hand, the negative relationship between high level of knowledge on LD and high acceptability of acaricides was unexpected. The relationship was also negative in Montérégie and in other intervention's models, but not statistically significant. A high level of knowledge was positively associated with higher levels of adoption of preventive behaviors against LD as performing tick checks, wearing protective clothing and putting tick repellent in another study [11]. Again, results of the qualitative analysis can help to understand this observation. In both regions, participants of the FGD had a higher level of knowledge than their respective population, as measured by Aenishaenslin in a previous study [41]: in Montérégie, $58 \%$ of the FGD participants had a good level of knowledge against only $15 \%$ in the survey, and in Neuchâtel, $86 \%$ of the FGD participants, compared to 51 $\%$ in the survey. This difference may be due to the recruitment process: FGD participants were recruited voluntarily by responding to a public invitation, so it is normal that this method selected individuals who were interested by the subject and who knew more about it than the population mean. Nevertheless, two important themes were omnipresent during discussions in Neuchâtel: 1) participants felt that individuals can protect themselves from LD by adopting preventive behaviors and should be responsible of their own protection, and then 2) they felt that LD risk was not high enough to use interventions with potential consequences on the environment. Indeed, our results suggest that a good level of knowledge may increase the perception of self-control on LD prevention, rather than increasing the acceptability of tick control interventions.

We found no statistical difference in the strength of association between high perceived efficacy of the intervention, risk perception, high level of knowledge on LD and high acceptability between both regions. This result is interesting given that the studied population contexts differed on several aspects, including their LD epidemiological situation, their history with the disease and their socio-cultural context. We could not find other published studies that measured such an association over the past. Further research conducted in other countries or regions where LD is present would be needed to support these findings.

The eight perceived issues identified to explain low acceptability of selected interventions have interesting similarities with the decision criteria identified by stakeholders involved in LD prevention and control in two previous studies conducted in Québec, Canada [38] and in Switzerland [39]. These studies respectively aimed at developing a multi-criteria decision model for LD prevention in Québec, and to adapt this model to the Swiss context, using a participatory approach with local stakeholders. These stakeholders participated in the elaboration of a list of important criteria to consider in order to prioritize LD preventive and control interventions, as 
well as a list of potential interventions. Most of the perceived issues explaining low acceptability find their equivalent in some of the criteria included in these two decision models. Conflict with personal values such as animal well-being and respect of natural environment, fear of environmental and other unknown consequences are issues addressed in the following criteria: "impacts on habitat", "impacts on wildlife" and "adverse health effects" [38]. Doubts about the feasibility of interventions are reflected by the inclusion of operational criteria such as the "complexity of the intervention's implementation" $[38,39]$ and the "durability of effects" [39]. Concerns with public costs are directly addressed with the criterion "cost to the public sector' $[38,39]$. Danger of disempowerment of the population is aligned with the criterion measuring the capacity of the intervention to raise the level of public awareness, which was added by Swiss stakeholders [39]. Moreover, the distinction made by participants to the FGD regarding large $v s$ small scales interventions is also taken into account in the decision models by separating some potential interventions (acaricide and landscaping) into small scale $v s$ large scale interventions in order to measure their impacts if they were implemented [38]. This observation suggests two interesting trends. First, issues expressed by individuals reflect community-level issues rather than only self-oriented considerations, such as adverse health effects on oneself or its close relatives. Second, these perceived issues are shared by stakeholders involved in LD management in public organisations and can be taken into account in the decision-making process by using tools such as multicriteria decision analysis [38].

This study has some limitations. First, this study design was cross-sectional and cannot presume of any causal relationships between factors associated with high acceptability in the quantitative analyses. Moreover, quantitative data were collected using a web panel and the sample cannot be considered as probabilistic. Nevertheless, distributions of gender, age and level of education of both studied samples were representative of demographic characteristics of Montérégie and Neuchâtel, as described in further details in Aenishaenslin et al. 2014 [41]. Another point to keep in mind is that several variables were measured with an ordinal scale in the survey, but were dichotomized for the analysis. This was done to allow a useful interpretation of the results in the public health context, to carry out multivariable logistic regression analyses and to maximise statistical power. But this methodological step results in a partial loss of information when compared to the raw survey data.

Regarding qualitative analysis, it is important to underline that the perceived issues identified to explain the low acceptability represent the perceptions of two small groups of participants, and may change over time according to contextual factors such as modifications in LD epidemiological situation or in the media attention toward this subject. The qualitative analysis was performed to explore perceived issues in the general public, but this approach cannot produce results that can be directly generalised to both regional populations. Also, FGD participants were recruited on a voluntary basis, and fewer participants manifested their interest to participate in Montérégie than in Neuchâtel (12 participants in Montérégie vs 22 in Neuchâtel). This can be understood by the difference in the levels of knowledge and risk perception between both regions [41], but may have affected the results. Moreover, it was noted that during the Montérégie FGD, participants had sometimes difficulties to express clear opinions on the acceptability of LD interventions given that they did not know enough about the disease, its consequences and individual preventive behaviors. This may reflect fluidity in the opinion expressed and, consequently, it would be worthwhile to follow-up on this study to assess variation in opinions as well as progress in the population's knowledge and perceptions since 2012.

\section{Conclusion}

This study strongly suggests that the perceived efficacy of public health interventions and LD risk perception in populations may be factors to consider in communication and knowledge translation efforts to increase the acceptability of tick control interventions when appropriate. Public health authorities may expect a higher level of reluctance to accept some of these interventions in populations with a higher level of knowledge about the disease, which may orient the consideration of different preventive strategies. In addition, low acceptability cannot be explained only by issues that may closely affect individuals such as immediate adverse health effects. Individuals who participated in this study expressed multiple issues related to LD management at the community level, such as possible impacts on environment and wildlife, high public costs and disempowerment of the population. These concerns are shared by decision-makers and can be integrated to the decision-making process by using tools such as multi-criteria decision analysis. Results of this study highlight the importance for decision-makers to account for regional socio-cognitive factors and perceived issues that may affect the acceptability of interventions in the target populations in order maximize the efficacy of interventions to prevent and control LD. Moreover, the study underlines the importance for decision-makers to be explicit about the public costs, the scale at which the intervention will be implemented, and its known impacts on ecosystems, when presenting an intervention to the target populations, as these aspects can modify its acceptability. 


\section{Competing interests}

The authors declare that they have no competing interests.

\section{Authors' contributions}

CA conceived and designed the study and drafted the manuscript. AR, PM and $L G$ helped in the elaboration of the questionnaires and organisation of focus groups. AR, PM, LG, FM, JPW and DB helped in the conception of the study. All authors read, revised and approved the final manuscript.

\section{Acknowledgements}

The authors would like to acknowledge Caroline Burri, Leonore Lovis and Liselore Roeslstra, as well as the University of Neuchâtel and the Direction de la santé publique de la Montérégie for their help in the organisation of the focus groups. Funding was provided by the Fonds de la recherche du Québec, the Public Health Agency of Canada, the 4P Training program in health promotion, prevention and public policy, and the Ministère de l'Éducation, des Loisirs et des Sports du Québec.

\section{Author details}

'Research Group on Epidemiology of Zoonoses and Public Health, Pavillon de la santé publique, Faculté de médecine vétérinaire, Université de Montréal, CP 5000, Saint-Hyacinthe, J2S 7C6 Québec, Canada. ²Laboratory for Foodborne Zoonoses, Public Health Agency of Canada, CP 5000, Saint-Hyacinthe, H2S 7C6 Québec, Canada. 'Laboratoire d'Eco-Épidémiologie, Institut de Biologie, Université de Neuchâtel, 11 Émile-Argand, CP 158, 2009 Neuchâtel, Switzerland. ${ }^{4}$ Institut national de santé publique du Québec, 1255 Beauregard, Lonqueuil, J4K 2M3 Québec, Canada. ${ }^{5}$ Group for Research in Decision Analysis (GERAD), 3000 Côte-Sainte-Catherine, Montréal H3T 2A7, Québec, Canada. ${ }^{6}$ Département de pathologie et microbiologie, Faculté de médecine vétérinaire, Université de Montréal, CP 5000, Saint-Hyacinthe, J2S 7C6 Québec, Canada.

Received: 1 October 2015 Accepted: 16 December 2015

\section{Published online: 05 January 2016}

\section{References}

1. Rizzoli A, Hauffe H, Carpi G, Vourc HG, Neteler M, Rosa R. Lyme borreliosis in Europe. Euro Surveill. 2011;16(27):1-8.

2. Steere AC, Coburn J, Glickstein L. The emergence of Lyme disease. J Clin Invest. 2004;113(8):1093-101.

3. Ogden $\mathrm{NH}$, Lindsay LR, Morshed M, Sockett PN, Artsob $H$. The emergence of Lyme disease in Canada. CMAJ. 2009;180(12):1221-4

4. Piesman J, Eisen L. Prevention of tick-borne diseases. Annu Rev Entomol. 2008;53:323-43.

5. Vazquez M, Muehlenbein C, Cartter M, Hayes EB, Ertel S, Shapiro ED Effectiveness of personal protective measures to prevent Lyme disease. Emerg Infect Dis. 2008;14(2):210-6.

6. Finch C, Al-Damluji MS, Krause PJ, Niccolai L, Steeves T, O'Keefe CF, et al. Integrated assessment of behavioral and environmental risk factors for Lyme disease infection on Block Island, Rhode Island. PLoS One. 2014:9(1):e84758.

7. Lane RS, Manweiler SA, Stubbs HA, Lennette ET, Madigan JE, Lavoie PE. Risk factors for Lyme disease in a small rural community in northern California. Am J Epidemiol. 1992;136(11):1358-68.

8. Smith G, Wileyto EP, Hopkins RB, Cherry BR, Maher JP. Risk factors for lyme disease in Chester County, Pennsylvania. Public Health Rep. 2001;116 Suppl 1:146-56.

9. Malouin R, Winch P, Leontsini E, Glass G, Simon D, Hayes EB, et al. Longitudinal evaluation of an educational intervention for preventing tick bites in an area with endemic lyme disease in Baltimore County, Maryland. Am J Epidemiol. 2003;157(11):1039-51.

10. Mowbray F, Amlot R, Rubin GJ. Ticking all the boxes? A systematic review of education and communication interventions to prevent tick-borne disease. Vector Borne Zoonotic Dis. 2012;12(9):817-25.

11. Aenishaenslin C, Michel P, Ravel A, Gern L, Milord F, Waaub JP, et al. Factors associated with preventive behaviors regarding Lyme disease in Canada and Switzerland: a comparative study. BMC Public Health. 2015:15:1539.

12. Herrington JE. Risk perceptions regarding ticks and Lyme disease: a national survey. Am J Prev Med. 2004;26(2):135-40.

13. Phillips CB, Liang MH, Sangha O, Wright EA, Fossel AH, Lew RA, et al. Lyme disease and preventive behaviors in residents of Nantucket Island, Massachusetts. Am J Prev Med. 2001;20(3):219-24.
14. Hallman W, Weinstein N, Kadakia S, Chess C. Precautions taken against Lyme disease at three recreational parks in endemic areas of New Jersey. Environ Behav. 1995;27(4):437-53.

15. Mowbray F, Amlot R, Rubin GJ. Predictors of protective behaviour against ticks in the UK: a mixed methods study. Ticks Tick Borne Dis. 2014;5(4):392-400

16. Valente SL, Wemple D, Ramos S, Cashman SB, Savageau JA. Preventive behaviors and knowledge of tick-borne illnesses: results of a survey from an endemic area. J Public Health Manag Pract. 2014;21(3):16-23.

17. Curran KL, Fish D, Piesman J. Reduction of nymphal Ixodes dammini (Acari: Ixodidae) in a residential suburban landscape by area application of insecticides. J Med Entomol. 1993;30(1):107-13.

18. Stafford 3rd KC, Ward JS, Magnarelli LA. Impact of controlled burns on the abundance of Ixodes scapularis (Acari: Ixodidae). J Med Entomol. $1998 \cdot 35(4) \cdot 510-3$

19. Schulze $T L$, Jordan RA. Potential influence of leaf litter depth on effectiveness of granular carbaryl against subadult lxodes scapularis (Acari: Ixodidae). J Med Entomol. 1995;32(2):205-8.

20. Schulze TL, Jordan RA, Hung RW. Suppression of subadult Ixodes scapularis (Acari: Ixodidae) following removal of leaf litter. J Med Entomol. 1995:32(5):730-3.

21. Samish M, Rehacek J. Pathogens and predators of ticks and their potential in biological control. Annu Rev Entomol. 1999:44:159-82.

22. Deblinger RD, Wilson ML, Rimmer DW, Spielman A. Reduced abundance of immature Ixodes dammini (Acari: Ixodidae) following incremental removal of deer. J Med Entomol. 1993;30(1):144-50.

23. Hoen AG, Rollen LG, Papero MA, Carroll JF, Daniels TJ, Mather TN, et al. Effects of tick control by acaricide self-treatment of white-tailed deer on host-seeking tick infection prevalence and entomologic risk for Ixodes scapularis-borne pathogens. Vector-Borne Zoonotic Dis. 2009:9(4):431-8.

24. Schulze TL, Jordan RA, Hung RW, Schulze CJ. Effectiveness of the 4-Poster passive topical treatment device in the control of Ixodes scapularis and Amblyomma americanum (Acari: Ixodidae) in New Jersey. Vector Borne Zoonotic Dis. 2009:9(4):389-400.

25. Brei B, Brownstein JS, George JE, Pound JM, Miller JA, Daniels TJ, et al. Evaluation of the United States Department of Agriculture northeast area-wide tick control project by meta-analysis. Vector Borne Zoonotic Dis. 2009;9(4):423-30.

26. Daniels TJ, Fish D, Falco RC. Evaluation of host-targeted acaricide for reducing risk of Lyme disease in southern New York state. J Med Entomol. 1991:28(4):537-43.

27. Deblinger RD, Rimmer DW. Efficacy of a permethrin-based acaricide to reduce the abundance of Ixodes dammini (Acari: |xodidae). J Med Entomol. 1991;28(5):708-11.

28. Mather TN, Ribeiro JM, Spielman A. Lyme disease and babesiosis: acaricide focused on potentially infected ticks. Am J Trop Med Hyg. 1987;36(3):609-14.

29. Stafford 3rd KC. Effectiveness of host-targeted permethrin in the control of Ixodes dammini (Acari: Ixodidae). J Med Entomol. 1991;28(5):611-7.

30. Stafford 3rd KC. Third-year evaluation of host-targeted permethrin for the control of Ixodes dammini (Acari: Ixodidae) in southeastern Connecticut. J Med Entomol. 1992:29(4):717-20.

31. Richer LM, Brisson D, Melo R, Ostfeld RS, Zeidner N, Gomes-Solecki M. Reservoir targeted vaccine against Borrelia burgdorferi: a new strategy to prevent Lyme disease transmission. J Infect Dis. 2014;209(12):1972-80.

32. Tsao K, Fish D, Galvani AP. Predicted outcomes of vaccinating wildlife to reduce human risk of Lyme disease. Vector Borne Zoonotic Dis. 2012;12(7):544-51.

33. Tsao Jl, Wootton JT, Bunikis J, Luna MG, Fish D, Barbour AG. An ecological approach to preventing human infection: vaccinating wild mouse reservoirs intervenes in the Lyme disease cycle. Proc Natl Acad Sci U S A. 2004;101(52):18159-64

34. Gomes-Solecki MJ, Brisson DR, Dattwyler RJ. Oral vaccine that breaks the transmission cycle of the Lyme disease spirochete can be delivered via bait. Vaccine. 2006:24(20):4440-9.

35. Llewellyn DM, Brazier A, Brown R, Cocker J, Evans ML, Hampton J, et al. Occupational exposure to permethrin during its use as a public hygiene insecticide. Ann Occup Hyg. 1996:40(5):499-509.

36. Alavanja MC, Hoppin JA, Kamel F. Health effects of chronic pesticide exposure: cancer and neurotoxicity. Annu Rev Public Health. 2004;25:155-97.

37. Sibbald B. Larvicide debate marks start of another West Nile virus summer. CMAJ. 2003;168(11):1455 
38. Aenishaenslin C, Hongoh V, Cisse HD, Hoen AG, Samoura K, Michel P, et al. Multi-criteria decision analysis as an innovative approach to managing zoonoses: results from a study on Lyme disease in Canada. BMC Public Health. 2013;13:897.

39. Aenishaenslin C, Gern L, Michel P, Ravel A, Hongoh V, Waaub JP, et al. Adaptation and evaluation of a multi-criteria decision analysis model for Lyme disease prevention. PLoS One. 2015;10(8):e0135171.

40. Gould LH, Nelson RS, Griffith KS, Hayes EB, Piesman J, Mead PS, et al. Knowledge, attitudes, and behaviors regarding Lyme disease prevention among Connecticut residents, 1999-2004. Vector Borne Zoonotic Dis. 2008;8(6):769-76.

41. Aenishaenslin C, Ravel A, Michel P, Gern L, Milord F, Waaub JP, et al. From Lyme disease emergence to endemicity: a cross sectional comparative study of risk perceptions in different populations. BMC Public Health. 2014;14(1):1298.

42. Léger Marketing. [http://leger360.com/en-ca/legerwebpanel.asp]. Accessed 1 Sept 2015.

43. Scherer R. PropCls: various confidence interval methods for proportions. 2014. http://cran.r-project.org/web/packages/PropCls/index.html. Accessed 1 Sept 2015.

44. Braun V, Clarke V. Using thematic analysis in psychology. Qual Res Psychol. 2006; $3(2): 77-101$

45. Vaismoradi $\mathrm{M}$, Turunen $\mathrm{H}$, Bondas $\mathrm{T}$. Content analysis and thematic analysis: Implications for conducting a qualitative descriptive study. Nurs Health Sci. 2013;15(3):398-405.

46. Tong A, Sainsbury P, Craig J. Consolidated Criteria for Reporting Qualitative Research (COREQ): a 32-item checklist for interviews and focus groups. International J Qual Health Care. 2007;19(6):349-57.

47. Ferrouillet $C$, Lambert L, Milord F. Consultation sur l'état actuel de la surveillance des zoonoses au Québec et son adéquation avec les changements climatiques et écologique. Institut national de santé publique du Québec. 2012. https://www.inspq.qc.ca/pdf/publications/1541_ConsulEtatActSurvZoonoQc AdeqChangClimEcolo.pdf. Accessed 1 Sept 2015

48. Nahimana I, Gern L, Peter O, Praz G, Moosmann Y, Francioli P. Epidemiology of Lyme borreliosis in French-speaking Switzerland. Schweiz Med Wochenschr. 2000;130(41):1456-61.

49. Moosmann Y, Brossard M, Raeber P, Gern L. Estimated Lyme borreliosis incidence in the canton of Neuchâtel (Switzerland). Bulletin de la Société neuchâteloise des Sciences Naturelles. 2012;132:47-56.

50. Conner M, Norman P. Predicting health behaviour. Whitby: Open University Press; 2005

\section{Submit your next manuscript to BioMed Central and we will help you at every step:}

- We accept pre-submission inquiries

- Our selector tool helps you to find the most relevant journal

- We provide round the clock customer support

- Convenient online submission

- Thorough peer review

- Inclusion in PubMed and all major indexing services

- Maximum visibility for your research

Submit your manuscript at www biomedcentral.com/submit

C Biomed Central 